\title{
Causal SNP regulating FAM13B expression identified for the Chr. $5 q 31$ atrial fibrillation susceptibility locus
}

Tchou: FAM13B gene and atrial fibrillation

Gregory Tchou ${ }^{1 *}$, Shamone Gore-Panter ${ }^{1,4^{*}}$, Jeffrey Hsu ${ }^{1 *}$, Fang Liu ${ }^{1 *}$, Nana Liu ${ }^{1}$, Christine S. Moravec $^{1,2}$, John Barnard ${ }^{3 \#}$, David R. Van Wagoner ${ }^{1,2 \#}$, Mina K. Chung ${ }^{1,2 \#}$, and Jonathan D. Smith ${ }^{1,2 \#}$

Departments of Cardiovascular \& Metabolic Scineces ${ }^{1}$, Cardiovascular Medicine ${ }^{2}$, and Quantitative Health Sciences ${ }^{3}$, Cleveland Clinic, Cleveland, OH, 44195, USA. Department of Biological, Geological and Environmental Science ${ }^{4}$, Cleveland State University, Cleveland, $\mathrm{OH}$

*, These authors contributed equally to this study.

\#, These authors are all corresponding authors

Address correspondence to Jonathan D. Smith, Box NC10, Cleveland Clinic, 9500 Euclid Avenue, Cleveland, $\mathrm{OH}, 44195$, USA

Phone: 216-444-2248, FAX: 216-444-9404, Email: smithj4@ccf.org

Word count of the manuscript:

Subject Codes: [132] Arrythmias-basic studies; [141] Functional genomics. 


\begin{abstract}
Rationale- Our prior RNA sequencing study found that FAM13B gene expression in human left atrial appendages was strongly associated with an atrial fibrillation (AF) susceptibilityassociated variant on chr. $5 \mathrm{q} 31$.

Objective- To identify the common genetic variant responsible for regulating FAM13B expression and the effect of FAM13B expression on cardiomyocyte gene expression in order to gain insight into the functional mechanism of the chr. $5 q 31$ AF susceptibility locus.

Methods and Results- By taking advantage of a smaller linkage disequilibrium block in African descent subjects and available chromatin conformation data, we identified the common single nucleotide polymorphism (SNP) rs17171731 as a candidate genetic variant controlling FAM13B gene expression in the left atrium. Functional analysis demonstrated that the AF risk allele of rs17171731 had less enhancer activity than the protective allele. Gel mobility shift studies determined that the risk allele bound to an additional protein that may function as a transcriptional repressor. Knockdown of $F A M 13 B$ expression in stem cell-derived human cardiomyocytes (iCM) altered the expression of $>1000$ genes and modified the sodium current, consistent with increased susceptibility to atrial fibrillation. Transfection of GFP tagged FAM13B into iCMs demonstrated expression on the plasma membrane and at the Z-disk.

Conclusions- The chr. 5q31 AF risk variant was identified as rs17171731, with the risk allele having less enhancer activity, leading to decreased expression of $F A M 13 B$, which resides on the plasma membrane and the Z-disk, and appears to play a role in the regulation of cardiomyocyte gene expression and the late sodium current.
\end{abstract}

Abbreviations:

GWAS, genome wide association study; AF, atrial fibrillation; SNP, single nucleotide polymorphism; eQTL, expression quantitative trait loci; LA, left atrium; RNAseq, next generation RNA sequencing; IncRNAs, long noncoding RNA; FDR, false discovery rate; AFR, atrial fibrillation rhythm at time of surgery; SR, sinus rhythm at time of surgery; SVA, surrogate variable analysis; VST, variance-stabilized transformation; MDS, multidimensional scaling; LAA, left atrial appendage; $A F / S R$, history of AF but in sinus rhythm at time of surgery; TSS, transcription start site; LD, linkage disequilibrium; iPSC, inducible pluripotent stem cell; $I_{N a}$, sodium current; $I_{\text {NaL, }}$ late sodium current. 
Atrial fibrillation (AF) is the most common human arrhythmia and has a strong heritable component. ${ }^{1}$ The most recent genome wide association studies (GWAS) for AF susceptibility identified 100 loci associated with AF. ${ }^{2,3}$. However, further work on each locus is necessary to identify the causal gene, the causal genetic variant, and the mechanism for association with AF. Since most AF GWAS SNPs, or those in strong linkage disequilibrium with them, do not alter the protein amino acid sequence, we hypothesize that most of these GWAS loci are regulatory, leading to changes in gene expression rather than protein structure. Our prior expression quantitative trait locus (eQTL) study utilized next generation RNA sequencing (RNAseq) and single nucleotide polymorphism (SNP) genotyping in human left atrial appendages to identify the cis genes whose expression are associated with the top SNP at the AF GWAS loci. ${ }^{4}$ We identified FAM13B as the gene whose expression is associated with the top GWAS SNP at chr. 5q31. Here, we describe how an eQTL analysis in a small African descent cohort led to the identification of a candidate SNP, rs17171731, in the chr. 5q31 AF GWAS locus that controls FAM13B gene expression. We validated this candidate SNP through reporter gene transfections, gel mobility shift assays, and gene editing in human pluripotent stem cells. Thus, the chr. 5q31 GWAS locus appears to be mediated by regulating the expression of FAM13B, a poorly characterized member of the rho GTP activating domain gene family. ${ }^{5,6}$ We then used siRNA to knockdown $F A M 13 B$ in stem cell-derived cardiomyocytes in order to investigate the mechanism by which decreased $F A M 13 B$ gene expression leads to increased AF risk. Knockdown of $F A M 13 B$ expression altered the expression of $>1000$ cardiomyocyte genes, including a subunit of the voltage-dependent sodium channel, which suggests possible mechanisms by which the genetic regulation of FAM13B could result in AF susceptibility. Expression of GFP-tagged FAM13B in stem cell derived cardiomyocytes s localized FAM13B protein to the plasma membrane and Z-disk.

\section{Methods}

\section{Human Left Atrial Tissue Samples and Processing}

Human left atrial appendage tissues were obtained from patients undergoing elective surgery to treat AF, valve disease, or other cardiac disorders. LA tissue specimens were also obtained from non-failing donor hearts not used for transplant. Demographics of this population were previously published. ${ }^{4}$ Total RNA was isolated for subsequent sequencing analysis. These methods are detailed in our prior publication. ${ }^{4}$

\section{Genomic DNA isolation and SNP microarray}

As previously described ${ }^{4}$, genomic DNA was genotyped using Illumina Hap550v3 and Hap610quad SNP microarrays. In order to derive genotypes in addition to those on the microarray, the SNP data was imputed to 1000 Genomes Project phase 2 to generate genotypes for 19 million SNPs, using the IMPUTE software. ${ }^{7}$ 


\section{RNA isolation, sequencing, and analysis}

As previously described ${ }^{4}, 100$-bp paired-end sequencing was performed on the Illumina HiSeq 2000 platform and multiplexed to 6 samples across two lanes. The sequence reads were mapped to the human genome to derive a digital count of the expression of genes, which were defined using the Ensembl gene catalog (version 71). Reads were quantile normalized, and gene counts for eQTL analysis were variance-stabilized transformed as previously described. ${ }^{4}$ eQTL analyses were performed separately for each racial group with beta-coefficients calculated as the additive effect of one allelic difference on $\log _{2}$ gene expression. Genetic multidimensional scaling (MDS) from SNP array genotyping were additionally calculated and added as covariates for the eQTL calculation. matrixeQTL ${ }^{8}$ was used to test associations between genotype and variance stabilized counts. The qvalue package was used to calculate false discovery rate (FDR) from the complete list of $p$ values. ${ }^{9}$

\section{Human stem cell culture and cardiac differentiation}

H9 human embryonic stem cells (WiCell) were cultured in TesR-E8 media (STEMCELL 05990) on plates coated with growth factor-reduced Matrigel (ThermoFisher CB-40230). After passaging, $\mathrm{H} 9$ cells were cultured in the presence of $10 \mu \mathrm{M}$ Y-27632 (Abcam) for 24-48 hours. Cardiac differentiation of $\mathrm{H} 9$ cells was conducted using the STEMdiff Cardiomyocyte Differentiation Kit (STEMCEII 05010).

\section{Reporter gene expression analysis}

26-mer oligonucleotides (Table 1) containing the FAM13B SNP rs17171731 reference or risk allele sequences were cloned into the luciferase reporter pT81luc. ${ }^{10}$ The luciferase reporter and the $\beta$-galactosidase expression vector $\mathrm{pCH} 110$ were transfected into $\mathrm{H} 9$ human embryonic stem cell-derived cardiomyocytes via electroporation (Nucleofector-II program A-23, Lonza VPH-5012). Cell lysates were prepared and analyzed for $\beta$-galactosidase and luciferase activities using the Dual Light Reporter Gene Assay system (ThermoFisher T1003).

\section{FAM13B electrophoretic mobility shift assays}

26-mer oligonucleotides containing the rs17171731 reference or risk allele sequences (Table 1) were labeled with $\mathrm{\gamma}^{32} \mathrm{P}$-ATP $(10 \mu \mathrm{Ci} / \mu \mathrm{L})$ using T4 polynucleotide kinase (NEB M0201) and annealed with complementary oligonucleotides in $10 \mathrm{mM}$ Tris, $1 \mathrm{mM}$ EDTA, $50 \mathrm{mM} \mathrm{NaCl}$. The resulting probes were combined on ice with human heart nuclear extract (Active Motif 36041), $1.25 \mu \mathrm{g}$ poly(dl dC) (Sigma-Aldrich P4929) and reaction buffer (final concentration $45 \mathrm{mM} \mathrm{KCl}, 1$ $\mathrm{mM} \mathrm{MgCl}_{2}, 15 \mathrm{mM}$ HEPES pH 7.9, $1 \mathrm{mM}$ DTT, 3\% Ficoll 400) in the presence or absence of unlabeled competitor oligonucleotides, then incubated at room temperature for 25 minutes before running on Novex 6\% DNA Retardation gels (ThermoFisher EC63655BOX). Dried Gels were exposed to X-ray film. We attempted to identify proteins bound to the probes using the 
following antibodies: ABI3, ATF4, MTF-1, POU2F1, POU2F2, SOX4, and SOX8 (One World Lab), as well as ZNF143 (R\&D Systems).

\section{IPSC-derived cardiomyocyte culture and siRNA knockdown of FAM13B}

Cardiomyocytes derived from human inducible pluripotent stem cells (iPSCs) (iCell cardiomyocytes, Cellular Dynamics International, Madison, WI) were cultured and siRNA knockdown was performed using Cellular Dynamics International protocols. In short, $1.5 \times 10^{5}$ cells were plated per well in fibronectin (Sigma)-coated 12-well plates. The cells were cultured in iCell cardiomyocyte maintenance media (Cellular Dynamics Int.) for seven days; beating was observed at about day six. Final concentrations of $50 \mathrm{nM} /$ well of control scramble siRNA (Thermo-Fisher/Ambion silencer select cat\#4390843) or FAM13B siRNA (ThermoFisher/Ambion cat\# 439242, id S27906) were transfected into iCell cardiomyocytes using the TransIT-TKO transfection reagent (Mirus Bio). The cells were incubated with the siRNA-reagent complexes for 48-96 hours followed by either cellular electrophysiology studies or total RNA isolation, cDNA preparation, qRT-PCR, and RNAseq (described above).

\section{Quantitative reverse transcriptase-polymerase chain reaction (qRT-PCR)}

Total RNA was isolated from the iCell cardiomyocytes after siRNA knockdown using the Cellular Dynamics International protocol (Total RNA protocol) and the RNeasy Micro Kit (Qiagen) with the following modification: from step 3 through 5, the reagent volumes were doubled to accommodate the increased well size. 100-250 $\mathrm{g}$ of RNA was reverse transcribed using SuperScript IV VILO ${ }^{\text {TM }}$ Master Mix (Thermo-Fisher/Invitrogen). $12.5 \mu$ l of the TaqMan ${ }^{\circledR}$ gene expression master mix (Applied Biosystems), $1.25 \mu$ of FAM-labeled FAM13B primer/probe mix (assay number HS00991421_M1, Thermo-Fisher), and 1.25 $\mu$ I VIC-labeled primer limited cardiac actin (ACTC1) primer/probe mix (assay number Hs00606316_m1, Thermo-Fisher) were mixed to create a master mix to be added to each sample. $15 \mu$ laliquots of master mix were dispensed into individual wells of a 96-well working plate along with $10 \mu$ l of undiluted cDNA. A $5 \mu \mathrm{l}$ aliquot of this working mixture was pipetted in triplicate to a 384-well assay plate using an epMotion (Eppendorf model 5070) automated pipetting robot. Real time PCR was performed using a Bio-RAD CRX thermocycler that is calibrated for FAM and VIC fluorescent probes. Thermal cycling was performed with a hot-start at $95^{\circ} \mathrm{C}$ for 10 minutes, followed by 40 cycles of $95^{\circ} \mathrm{C}$ for 15 seconds and $60^{\circ} \mathrm{C}$ for 60 seconds. The $\Delta C(t)$ values for FAM13B expression levels were calculated relative to ACTC1. $\triangle \triangle \mathrm{C}(\mathrm{t})$ values were used to calculate the decrease of FAM13B expression and the results were converted to base 10 values by calculating $2^{-\Delta \Delta C(t)}$.

\section{Cellular electrophysiology methods}

Patch clamp studies of FAM13B knockdown cells focused on measurement of sodium currents (INa) in scramble and FAM13B-siRNA treated iCell cardiomyocytes. Cells were replated at low 
density 24 hours after siRNA treatment (non-touching), and electrophysiology studies were performed 48 to 96 hours after transfection. Sodium currents were recorded at room temperature using whole cell clamp techniques. Pipettes (Corning 8161 thin-walled glass, 1.5-3 $\mathrm{M} \Omega$ ) were filled with a solution containing $135 \mathrm{mM} \mathrm{CsCl} 2,10 \mathrm{mM} \mathrm{NaCl}, 2 \mathrm{mM} \mathrm{CaCl}$, $5 \mathrm{mM}$ EGTA, $10 \mathrm{mM}$ HEPES, $5 \mathrm{mM} \mathrm{MgATP}$, and titrated to $\mathrm{pH} 7.2$ with $\mathrm{CsOH}$. The cells were continuously superfused with an extracellular solution containing: $50 \mathrm{mM} \mathrm{NaCl}, 1.8 \mathrm{mM} \mathrm{CaCl}, 1$ $\mathrm{mM} \mathrm{MgCl}_{2}, 110 \mathrm{mM} \mathrm{CsCl}, 10 \mathrm{mM}$ glucose, $10 \mathrm{mM}$ HEPES, $1 \mu \mathrm{M}$ nifedipine (added fresh daily), $\mathrm{pH}$ 7.4. Currents were recorded with an Axopatch $1 \mathrm{C}$ amplifier controlled with pClamp 8.1 software (Molecular Devices). After seal formation and patch rupture, whole cell access resistance was 2-5 M $\Omega$. To maintain cell viability, resting potential was held at $-60 \mathrm{mV}$, with more negative steps given 100 ms before depolarizing steps used to record sodium currents. Voltage clamp protocols are shown in Figure 4 A,C,F. Current amplitudes recorded with the steady-state inactivation protocol (Figures 4C,D) were fit with a Boltzmann curve in Clampfit 8.1 (pClamp, Molecular Devices). To avoid time-dependent changes in siRNA exposure, data were obtained from similar numbers of scramble and siRNA-treated cells on each day. Data are reported as mean \pm standard error of the mean. All electrophysiologic comparisons between scramble and FAM13B-siRNA treated cells were evaluated using a 2 tailed T-test or a 2-way ANOVA as indicated.

\section{Analysis of FAM13B-GFP expression}

The FAM13B-EGFP fusion protein expression vector was constructed by VectorBuilder Inc (\#VB190426-1100mup). The elongation factor 1 $\alpha$ short (EFS) promoter drives the expression of the human FAM13B coding sequence[NM_016603.3] fused at its C-terminus to the eGFP coding sequence, with a Neomycin resistance gene inserted downstream of FAM13B-EGFP sequence connected by T2A Linker.. iCell Cardiomyocytes (Cellular Dynamics) were plated at a density of $5 \times 10^{4}$ cells per well into $0.1 \%$ Gelatin treated glass-bottom 96 -well plate according to the manufacturer's instruction. The cells were maintained in iCell Cardiomyocytes Maintenance medium. 48 hours later, the cells were transfected with EFS-FAM13B-EGFP plasmid using ViaFect transfection Reagent (Promega Corporation) at a 4:1 reagent-to-DNA ratio (vol:wt) and $0.1 \mu \mathrm{g}$ plasmid DNA per well. Four to six days later, the cardiomyocytes were fixed with $10 \%$ Neutral Buffered Formalin for $10 \mathrm{~min}$ at room temperature. After incubation with $0.1 \%$ Triton $X 100$ for $20 \mathrm{~min}$ to permeabilize the cell membrane, the cells were washed and incubated with Casein Blocker (Thermo Fisher Scientific) for 1 hour at room temperature. The cells were incubated with mouse anti $\alpha$-actinin primary antibody (1:500 dilution, \#A7811, Sigma Aldrich) at $4^{\circ} \mathrm{C}$ overnight, and then with Alexa Fluor 568 labeled goat anti-mouse IgG secondary antibody (1:500 dilution, \#A11004, Thermo Fisher Scientific) for 2 hours at room temperature. Nuclei were counterstained with Hoechst 33342 for $5 \mathrm{~min}$ and epifluorescent micrographs were obtained. 


\section{Data Access}

Variance normalized gene expression levels for each subject are available in the GEO database, accession number GSE69890. LAA eQTL data can be obtained on our web browser:

http://afeqtl.lerner.ccf.org/.

\section{Results}

\section{African descent eQTL analysis for FAM13B gene expression}

Our published left atrial appendage RNAseq-eQTL analysis focused on 235 subjects with selfreported European ancestry as well as 30 African American subjects whose ancestry was verified by SNP array principal component analysis, to be admixed or clustered closely with African reference samples. ${ }^{4}$ In the European ancestry subjects, we showed that the GWAS SNP at the chr. 5q31 locus, rs2040862, was a strong eQTL for expression of the FAM13B gene ( $p=$ $\left.7 \times 10^{-30}\right)$. However, this SNP is in strong linkage disequilibrium (LD) with many other SNPs that have similar eQTL p-values. ${ }^{4}$ Although our African American cohort was small, we were able to detect a significant eQTL for FAM13B expression. The rs17171731 SNP, located $20 \mathrm{~kb}$ upstream of the FAM13B transcription start site, stood out with an eQTL p-value $\sim 10$-fold stronger than the other SNPs in this region (Figure $1 A, B ; p=2.63 \times 10^{-5}$ ). rs17171731 is in high LD with the GWAS SNP in our European ancestry subjects $\left(r^{2}=0.96\right)$. Note, $r s 17171731$ is not included in the 1000 Genomes phase 3 database, but it was present in the phase 1 database used for imputation.

\section{Functional studies of the FAM13B candidate causal SNP}

Based on the cis-eQTL analysis (Figure 1A, B) and DNasel hypersensitivity in human fetal heart (Figure 1C), we identified rs17171731 as the top candidate causal SNP regulating FAM13B gene expression. In order to detect enhancer activity in this region, we subcloned 26-mer oligos containing the reference or risk alleles of rs17171731 (Table 1) into a luciferase reporter plasmid driven by a minimal viral thymidine kinase (TK) promoter (pT81luc). After transfection into cardiomyocytes differentiated from $\mathrm{H} 9$ embryonic stem cells, we observed that the risk allele had weak enhancer activity, and the reference allele had 3.3-fold stronger activity vs. the risk allele $(p<0.001$, Figure $2 A)$.

We then performed electromobility shift assays using ${ }^{32} \mathrm{P}$-labeled 26 mer oligos surrounding the rs17171731 SNP (Figure 2B). Incubation of the reference allele probe with human heart nuclear extract yielded one strongly shifted fragment (Band 1), while the risk allele yielded both Band 1 and a smaller shifted fragment (Band 2). Band 1 was effectively competed off of both probes with excess unlabeled reference or risk oligos; however, Band 2 was competed off more effectively with the risk vs. reference oligo. Using a different lot of heart nuclear extract that 
had more prominent Band 2 vs. Band 1 shifts, along with the risk allele probe, we performed competition studies using nine unlabeled oligos (Table 1 ) with single bp changes extending \pm 4 bp from the rs17171731 SNP site (Figure 2C). We determined that the central sequence motif required for the Band 2 shift was TGCA, with the rs17171731 risk allele representing the $C$ of this motif. We searched the JASPAR transcription factor motif database ${ }^{11}$ for TGCA binding factors with scores $>93.5$ and narrowed our list to those that were expressed in LA based on our RNAseq data ${ }^{4}$, which yielded the following eight factors: ATF4, POU2F1, SOX4, ZNF143, SOX8, $A B I 3, M T F 1$, and POU2F2. Commercially available antibodies against these proteins did not supershift or block Band 2 formation (data not shown); thus, we did not identify the heart nuclear protein(s) responsible for the Band 2 shift, which presumably mediate the silencing activity of the rs17171731 risk allele.

FAM13B (prior gene name C5ORF5 ${ }^{5}$ ) is a member of the Rho-GAP gene family. In silico analysis of the FAM13B Rho-GAP domain indicates that two highly conserved putative GTPase catalytic residues are altered in the FAM13B protein, potentially yielding a non-catalytic protein. ${ }^{5}$ To begin to evaluate the mechanism whereby FAM13B impacts atrial arrhythmogenesis, RNAseq and patch clamp studies were performed to assess the effect of FAM13B siRNA-transfection vs. a scrambled siRNA on cardiomyocytes derived from iPSCs. Four days after FAM13B siRNA transfection, we observed $77 \%$ knockdown of FAM13B mRNA expression by qPCR (Figure 3A). RNAseq analysis of the FAM13B and scrambled siRNA treated cells revealed that expression of 1156 genes was significantly altered after FAM13B knockdown (FDR adjusted $p<0.1, n=3$ per condition, Supplemental Table 1), and the top 20 most significant changes in gene expression are shown in Table 2. While expression levels of prominent atrial ion channel pore subunits were not significantly impacted by $F A M 13 B$ knockdown, expression of $S C N 2 B$, which encodes a beta-subunit of the cardiac sodium channel, was the $11^{\text {th }}$ strongest regulated transcript, and it was down regulated 2.2-fold by FAM13B knockdown (Table 2). Ingenuity Pathway analysis revealed that $S C N 2 B$ was a member of the second strongest empirical network associated with FAM13B knockdown (Figure 3B).

Patch clamp studies of $F A M 13 B$ knockdown cells focused on measurement of sodium current $\left(I_{\mathrm{Na}}\right.$ ) density (Figure 4A,B), steady state inactivation (Figure 4C,D) and late sodium current ( $I_{\mathrm{NaL}}$, Figure $4 E, F)$. FAM13B knockdown induced loss of $S C N 2 B$ did not significantly impact the peak sodium current density (Figure 4B). FAM13B knockdown increased the $\mathrm{V}_{1 / 2}$ for the steady state inactivation of the $I_{N a}$ by $2.7 \mathrm{mV}$ (Figure 4D), and although this effect did not reach $\mathrm{p}<0.05$ by $\mathrm{t}$ test, a 2-way ANOVA test showed a significant difference between the curves $(p=0.002$ for interaction between treatment and voltage). Cell capacitance was not significantly different between groups (KD: $39.5 \pm 6.5 \mathrm{pF}$ vs. SCR $36.4 \pm 4.5 \mathrm{pF}$ ). Late sodium current density (I $\mathrm{I}_{\mathrm{NaL}}$ ) was evaluated using a ramp clamp protocol (Figure $4 \mathrm{E}$ ). There was a tendency for $I_{\text {NaL }}$ density to be larger in the $F A M 13 B$ knockdown cells compared to the scramble $(-1.73 \pm 0.33$ vs. $-1.24 \pm 0.13$ 
$\mathrm{pA} / \mathrm{pF}, \mathrm{p}=0.20$ ). When subdivided by cell capacitance above and below the median value (37.7 $\mathrm{pF}$ ), the difference in $\mathrm{I}_{\mathrm{NaL}}$ density between knockdown and scramble groups was significant for the smaller capacitance cells ( $p=0.0443)$, but not for the larger capacitance cells $(p=0.879)$. The ratio of $I_{\mathrm{NaL}} /$ peak $I_{\mathrm{Na}}$ was significantly increased in the FAM13B knockdown cells, regardless of cell capacitance (Figure 4F, p=0.025).

To gain more insight into FAM13B mechanism of action, we transfected different cell types with a FAM13B-GFP fusion protein construct. Upon transfection into non-cardiomyocyte HEK293 and human iPS cells, we observed a few GFP+ cells two days after transfection, but these cells were compact, round, and poorly attached, and had died by day four, such that no GFP+ cells remained on the dish. However, after transfection into iCell cardiomyocytes, we observed robust GFP+ staining on the plasma membrane and in sarcomeres (Figure 5). We used antibodies directed to the Z-disc ( $\alpha$-actinin), the sarcoplasmic reticulum (serca2) and we observed the better co-localization with $\alpha$-actinin (Figure 5 ).

\section{Discussion}

In our prior LAA RNAseq analysis, the second strongest AF GWAS SNP eQTL was for FAM13B at a locus previously attributed to WNT8A. ${ }^{4}$ At this locus, the minor allele of rs 2040862 is the AF risk allele, and this allele is associated with decreased expression of FAM13B. The FAM13B gene encodes an uncharacterized protein containing a Rho GTPase activation protein (RhoGAP) domain. ${ }^{5}$ The eQTL plots for LAA FAM13B expression in European and African American descent subjects and a DNasel hypersensitivity peak map in fetal heart tissue allowed us to identify rs17171731 as a top candidate functional SNP. Our reporter gene transfection into iPS derived cardiomyocytes show that rs17171731 is a causal variant, with the risk allele having decreased enhancer activity, in the same direction as observed in human LAA tissue. A different SNP at chr. 5q31 (rs1004989) that was previously associated with the electrocardiographic QTinterval was found to be a significant eQTL for FAM13B expression in left ventricle tissue samples $^{12}$; however, rs1004989 is not in LD with the best left atrial FAM13B eQTL SNP (rs17171731), perhaps illuminating tissue-specific regulatory variants controlling FAM13B gene expression.

To begin to assess the functional role of $F A M 13 B$, we used siRNA knockdown in human cardiomyocytes differentiated from iPSCs. RNAseq analysis of the siRNA knockdown cells showed a striking pattern with extensive co-regulation with more than 1000 other genes, suggesting the FAM13B may be a hub gene that plays a role in regulating LA physiology. Pathway analysis found a strong empirical network that included $S C N 2 B$, a subunit of the cardiac sodium channel, which we identified as one of the most down-regulated genes. In ventricular cardiomyocytes from normal and heart failure dogs, virally delivered siRNA knockdown of SCN2B was associated with increased late sodium current $\left(I_{\mathrm{NaL}}\right) .{ }^{13} \mathrm{I}_{\mathrm{NaL}}$, resulting 
from either genetic or environmental influences, has been associated with action potential prolongation in heart failure, and both $\mathrm{I}_{\mathrm{NaL}}{ }^{14}$ and mutations in SCN2B have been associated with risk of AF. ${ }^{15}$ Results of our electrophysiology studies in the FAM13B knock-down iPSC-derived cardiomyocytes were similar to the effects of SCN2B knockdown in canine ventricular cardiomyocytes. ${ }^{13}$ We noted trends toward a positive shift in the steady state inactivation of the sodium current (Figure 4D) and an increased density of the slowly inactivating sodium current (Figure 4E), resulting in a significantly increased fraction of $I_{\text {NaL }} /$ peak $I_{\text {Na }}$ (Figure $4 F$ ). In our study, peak $I_{\mathrm{Na}}$ was not different between groups (Figure $4 \mathrm{~B}$ ), but $I_{\text {NaL }}$ was increased in smaller (likely atrial and nodal) cells to a greater extent than in the larger capacitance cells. Sodium currents are not identical in atrial and ventricular cardiomyocytes, likely due to differences in sodium channel subunit composition and cellular architecture.

The determinants of peak and late sodium current density are complex, with roles for channel trafficking, CaMKII phosphorylation, subunit interactions, oxidative stress and metabolic status suggested as potential modifiers. We suggest that increased $I_{\text {NaL, }}$ which indirectly modifies intracellular calcium levels as a result of sodium-calcium exchanger activity, may represent one of the pathways whereby a genetically determined reduction in FAM13B abundance increases risk of AF. Other sodium channel (SCN5A) gain of function mutations that increase sodium influx have also been associated with increased risk of cardiac arrhythmia. ${ }^{16,17}$ Our studies suggest that inhibition of $I_{\text {NaL }}$ with new or available drugs, such as ranolazine, may reduce $A F$ risk in patients carrying the $F A M 13 B$ risk allele.

Expression of a FAM13B-GFP fusion protein in iCell cardiomyocytes demonstrated localization both in sarcomeres and on the plasma membrane (Figure 5). The sarcomere staining overlaps or was adjacent to the Z-disc marker protein $\alpha$-actinin. This pattern may be due to FAM13B located on the Z-disk and/or the T-tubule, which connects the plasma membrane with deep invaginations near the Z-disk. Using the Gene Onloogy list for genes associated with the Z-disk in mice, we found the following other AF GWAS genes ${ }^{2}$ that share this association: CASQ2, CFL2, FBXO32, KCNN2, MYH7, MYO18B, MYOZ1, SYNE2, SYNPO2L, and TTN

Further understanding of the mechanism by which low expression of FAM13B, along with its binding partners and associated pathways, can predispose to AF susceptibility may illuminate targets for novel therapies. Thus, FAM13B is a potential target for drug development to prevent or treat AF.

\section{Acknowledgements}

This work was supported by the National Institutes of Health grant RO1 HL 111314 to MKC, DVW, JB, and JDS, an American Heart Association Strategically Focused Research Network grant 18SFRN34110067 to MKC, DVW, JB and JDS, the NIH National Center for Research Resources for 
Case Western Reserve University and Cleveland Clinic Clinical and Translational Science Award UL1-RR024989, the Cleveland Clinic Department of Cardiovascular Medicine philanthropy research funds, and the Tomsich Atrial Fibrillation Research Fund. JH was supported by the National Institutes of Health training grant T32 GM 088088. JDS was supported by the Geoffrey Gund Endowed Chair for Cardiovascular Research.

\section{Disclosure Declaration}

The authors declare no disclosures for this work. 


\section{References}

1. Ellinor PT, Yoerger DM, Ruskin JN, MacRae CA. Familial aggregation in lone atrial fibrillation. Hum Genet. 2005;118:179-184.

2. Roselli C, Chaffin MD, Weng L-C, Aeschbacher S, Ahlberg G, Albert CM, Almgren $P$, Alonso A, Anderson CD, Aragam KG, Arking DE, Barnard J, Bartz TM, Benjamin EJ, Bihlmeyer NA, Bis JC, Bloom HL, Boerwinkle E, Bottinger EB, Brody JA, Calkins H, Campbell A, Cappola TP, Carlquist J, Chasman DI, Chen LY, Chen Y-DI, Choi E-K, Choi SH, Christophersen IE, Chung MK, Cole JW, Conen D, Cook J, Crijns HJ, Cutler MJ, Damrauer SM, Daniels BR, Darbar D, Delgado G, Denny JC, Dichgans M, Dörr M, Dudink EA, Dudley SC, Esa N, Esko T, Eskola M, Fatkin D, Felix SB, Ford I, Franco OH, Geelhoed B, Grewal RP, Gudnason V, Guo X, Gupta N, Gustafsson S, Gutmann R, Hamsten A, Harris TB, Hayward C, Heckbert SR, Hernesniemi J, Hocking LJ, Hofman A, Horimoto ARVR, Huang J, Huang PL, Huffman J, Ingelsson E, Ipek EG, Ito K, Jimenez-Conde J, Johnson R, Jukema JW, Kääb S, Kähönen M, Kamatani Y, Kane JP, Kastrati A, Kathiresan S, Katschnig-Winter $P$, Kavousi M, Kessler T, Kietselaer BL, Kirchhof P, Kleber ME, Knight S, Krieger JE, Kubo M, Launer L, Laurikka J, Lehtimäki T, Leineweber K, Lemaitre RN, Li M, Lim HE, et al. Multi-ethnic genomewide association study for atrial fibrillation. Nat Genet. 2018;50:1225-1233.

3. Nielsen JB, Thorolfsdottir RB, Fritsche LG, Zhou W, Skov MW, Graham SE, Herron TJ, McCarthy S, Schmidt EM, Sveinbjornsson G, Surakka I, Mathis MR, Yamazaki M, Crawford RD, Gabrielsen ME, Skogholt AH, Holmen OL, Lin M, Wolford BN, Dey R, Dalen H, Sulem P, Chung JH, Backman JD, Arnar DO, Thorsteinsdottir U, Baras A, O'Dushlaine C, Holst AG, Wen X, Hornsby W, Dewey FE, Boehnke M, Kheterpal S, Mukherjee B, Lee S, Kang HM, Holm H, Kitzman J, Shavit JA, Jalife J, Brummett CM, Teslovich TM, Carey DJ, Gudbjartsson DF, Stefansson K, Abecasis GR, Hveem K, Willer CJ. Biobank-driven genomic discovery yields new insight into atrial fibrillation biology. Nat Genet. 2018;1234-1239.

4. Hsu J, Gore-panter S, Tchou G, Castel L, Lovano B, Moravec CS, Pettersson GB, Eric E, Gillinov AM, Mccurry KR, Smedira NG, Barnard J, Wagoner DR Van, Chung MK. Genetic Control of Left Atrial Gene Expression Yields Insights into the Genetic Susceptibility for Atrial Fibrillation. Circ Genom Precis Med. 2018;11:e00210.

5. Lai F, Godley LA, Fernald AA, Orelli BJ, Pamintuan L, Zhao N, Le Beau MM. cDNA cloning and genomic structure of three genes localized to human chromosome band $5 q 31$ encoding potential nuclear proteins. Genomics. 2000;70:123-30.

6. Cohen M, Reichenstein M, der Wind A, Heon-Lee J, Shani M, Lewin HA, Weller Jl, Ron M, Seroussi E. Cloning and characterization of FAM13A1-a gene near a milk protein QTL on BTA6: evidence for population-wide linkage disequilibrium in Israeli Holsteins. Genomics. 2004;84:374-383.

7. Marchini J, Howie B, Myers S, McVean G, Donnelly P. A new multipoint method for genome-wide association studies by imputation of genotypes. Nat Genet. 2007;39:906-13.

8. Shabalin A a. Matrix eQTL: ultra fast eQTL analysis via large matrix operations. Bioinformatics. 2012;28:1353-8.

9. Storey JD, Tibshirani R. Statistical significance for genomewide studies. Proc Natl Acad Sci. 2003;100:9940-9945. 
10. Nordeen SK. Luciferase reporter gene vectors for analysis of promoters and enhancers. Biotechniques. 1988;6:454-458.

11. Mathelier A, Fornes O, Arenillas DJ, Chen C, Denay G, Lee J, Shi W, Shyr C, Tan G, Worsley-Hunt R, others. JASPAR 2016: a major expansion and update of the open-access database of transcription factor binding profiles. Nucleic Acids Res. 2016;44:D110-D115.

12. Arking DE, Pulit SL, Crotti L, der Harst P, Munroe PB, Koopmann TT, Sotoodehnia N, Rossin EJ, Morley M, Wang X, others. Genetic association study of QT interval highlights role for calcium signaling pathways in myocardial repolarization. Nat Genet. 2014;46:826-836.

13. Mishra S, Undrovinas NA, Maltsev VA, Reznikov V, Sabbah HN, Undrovinas A. Post-transcriptional silencing of SCN1B and SCN2B genes modulates late sodium current in cardiac myocytes from normal dogs and dogs with chronic heart failure. Am J Physiol Circ Physiol. 2011;301:H1596-H1605.

14. Fischer TH, Herting J, Mason FE, Hartmann N, Watanabe S, Nikolaev VO, Sprenger JU, Fan P, Yiao L, Popov A-F, others. Late INa increases diastolic SR-Ca2+-leak in atrial myocardium by activating PKA and CaMKII. Cardiovasc Res. 2015;107:184-196.

15. Watanabe H, Darbar D, Kaiser DW, Jiramongkolchai K, Chopra S, Donahue BS, Kannankeril PJ, Roden DM. Mutations in sodium channel $\$ \beta \$ 1$-and $\$ \beta \$ 2$-subunits associated with atrial fibrillation. Circ Arrhythmia Electrophysiol. 2009;2:268-275.

16. Tian X-L, Yong SL, Wan X, Wu L, Chung MK, Tchou PJ, Rosenbaum DS, Van Wagoner DR, Kirsch GE, Wang $Q$. Mechanisms by which SCN5A mutation N1325S causes cardiac arrhythmias and sudden death in vivo. Cardiovasc Res. 2004;61:256-267.

17. Blana A, Kaese S, Fortmüller L, Laakmann S, Damke D, van Bragt K, Eckstein J, Piccini I, Kirchhefer $U$, Nattel S, others. Knock-in gain-of-function sodium channel mutation prolongs atrial action potentials and alters atrial vulnerability. Hear Rhythm. 2010;7:1862-1869. 
Table 1. Oligonucleotides used for FAM13B enhancer screen and gel mobility shift assays.

\begin{tabular}{|c|c|c|}
\hline & sense & antisense \\
\hline FAM13b ref allele & ACCTTGGACCCATTCCATCCAGTATC & TGGAACCTGGGTAAGGTAGGTCATAG \\
\hline FAM13b risk allele & ACCTTGGACCCATTGCATCCAGTATC & TGGAACCTGGGTAA $\underline{\mathbf{C}}$ GTAGGTCATAG \\
\hline FAM13b risk $-4 C>G$ & ACCTTGGACCGATTGCATCCAGTATC & TGGAACCTGGC्CAACGTAGGTCATAG \\
\hline FAM13b risk $-3 \mathrm{~A}>\mathrm{T}$ & ACCTTGGACCCTTTGCATCCAGTATC & TGGAACCTGGGÄAACGTAGGTCATAG \\
\hline FAM13b risk $-2 \mathrm{~T}>\mathrm{A}$ & ACCTTGGACCCAATGCATCCAGTATC & TGGAACCTGGGT襾GTAGGTCATAG \\
\hline FAM13b risk $-1 T>A$ & ACCTTGGACCCATAGGATCCAGTATC & TGGAACCTGGGTA $\underline{\mathbf{T} C G T A G G T C A T A G}$ \\
\hline FAM13b risk $+1 C>G$ & ACCTTGGACCCATTGGATCCAGTATC & TGGAACCTGGGTAACĆTAGGTCATAG \\
\hline FAM13b risk $+2 \mathrm{~A}>\mathrm{T}$ & ACCTTGGACCCATTGCTTCCAGTATC & TGGAACCTGGGTAACGAAGGTCATAG \\
\hline FAM13b risk $+3 \mathrm{~T}>\mathrm{A}$ & ACCTTGGACCCATTGCAACCAGTATC & TGGAACCTGGGTAACGT金GGTCATAG \\
\hline FAM $13 \mathrm{~b}$ risk $+4 \mathrm{C}>\mathrm{G}$ & ACCTTGGACCCATTGCATGCAGTATC & TGGAACCTGGGTAACGTACGTCATAG \\
\hline
\end{tabular}


Table 2. Twenty most significantly regulated genes after FAM13B knockdown.

\begin{tabular}{|c|c|c|c|c|c|c|c|}
\hline \# & Gene Symbol & Ensembl GenelD & $\begin{array}{l}\text { Gene } \\
\text { Length } \\
\text { (bp) }\end{array}$ & $\begin{array}{l}\text { Log2 Fold Change } \\
\text { (FAM13B siRNA vs. } \\
\text { scr siRNA) }\end{array}$ & $\begin{array}{c}\text { Average Expression } \\
\text { (Log2 counts/ } \\
\text { million) }\end{array}$ & P-Value & adj. P-Value \\
\hline 1 & EPPK1 & ENSG00000261150 & 7273 & 1.51 & 7.56 & 9.99E-07 & 3.09E-03 \\
\hline 2 & TBC1D8 & ENSG00000204634 & 4147 & -0.97 & 6.49 & $1.12 \mathrm{E}-06$ & $3.09 E-03$ \\
\hline 3 & HDDC2 & ENSG00000111906 & 1615 & -1.14 & 4.83 & $1.12 \mathrm{E}-06$ & $3.09 E-03$ \\
\hline 4 & MYH11 & ENSG00000133392 & 6942 & 1.84 & 3.25 & $4.32 \mathrm{E}-07$ & 3.09E-03 \\
\hline 5 & SLC4A4 & ENSG00000080493 & 8010 & -1.03 & 5.33 & $1.74 \mathrm{E}-06$ & $3.83 E-03$ \\
\hline 6 & TCAP & ENSG00000173991 & 963 & -1.06 & 8.37 & $5.38 \mathrm{E}-06$ & $7.46 \mathrm{E}-03$ \\
\hline 7 & RASD1 & ENSG00000108551 & 1746 & -0.88 & 4.64 & 5.99E-06 & $7.46 \mathrm{E}-03$ \\
\hline 8 & FAM120B & ENSG00000112584 & 3239 & -0.90 & 4.33 & $6.74 \mathrm{E}-06$ & $7.46 \mathrm{E}-03$ \\
\hline 9 & SLC41A1 & ENSG00000133065 & 4854 & -0.93 & 6.40 & $7.78 \mathrm{E}-06$ & $7.46 \mathrm{E}-03$ \\
\hline 10 & ACE2 & ENSG00000130234 & 3507 & 1.30 & 6.56 & $8.12 \mathrm{E}-06$ & $7.46 \mathrm{E}-03$ \\
\hline 11 & SCN2B & ENSG00000149575 & 4922 & -1.14 & 4.31 & 7.33E-06 & $7.46 \mathrm{E}-03$ \\
\hline 12 & SCML1 & ENSG00000047634 & 2911 & -1.55 & 3.09 & $4.85 \mathrm{E}-06$ & $7.46 \mathrm{E}-03$ \\
\hline 13 & PDE4DIP & ENSG00000178104 & 12539 & -1.15 & 10.00 & $9.20 \mathrm{E}-06$ & $7.80 \mathrm{E}-03$ \\
\hline 14 & XRCC4 & ENSG00000152422 & 1704 & 0.90 & 7.20 & $1.21 \mathrm{E}-05$ & $8.41 \mathrm{E}-03$ \\
\hline 15 & FAM13B & ENSG00000031003 & 5624 & -2.17 & 5.40 & 1.17E-05 & 8.41E-03 \\
\hline 16 & MT1X & ENSG00000187193 & 404 & 1.04 & 3.28 & $1.22 \mathrm{E}-05$ & $8.41 \mathrm{E}-03$ \\
\hline 17 & KRT80 & ENSG00000167767 & 3894 & 0.79 & 4.74 & $1.38 \mathrm{E}-05$ & $8.64 \mathrm{E}-03$ \\
\hline 18 & CASQ2 & ENSG00000118729 & 2702 & 1.51 & 9.30 & $1.55 \mathrm{E}-05$ & 8.64E-03 \\
\hline 19 & SLC40A1 & ENSG00000138449 & 3355 & -1.29 & 5.68 & $1.60 \mathrm{E}-05$ & $8.64 \mathrm{E}-03$ \\
\hline 20 & DRAM2 & ENSG00000156171 & 1886 & 0.71 & 5.18 & $1.74 \mathrm{E}-05$ & 8.64E-03 \\
\hline
\end{tabular}


Figure 1.
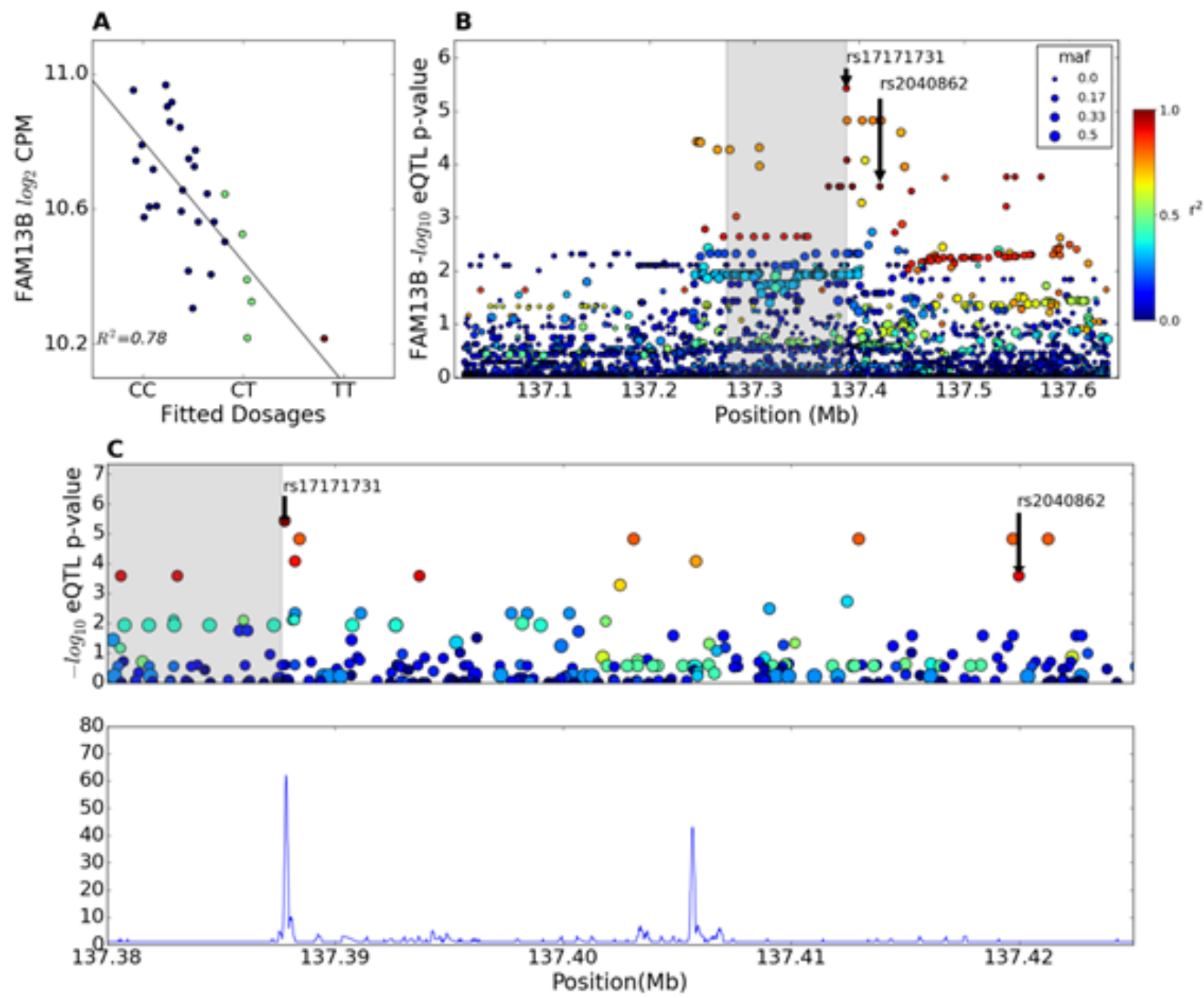

Figure 1. FAM13B cis-eQTL. A. Partial regression plot showing the effect of rs17171731 on LA expression of the $F A M 13 B$ gene in African American subjects. Blue dots are subjects homozygous for the major allele, green dots are heterozygotes, and red dots are homozygous for the minor allele $\left(r^{2}=0.78\right.$, $q$-value $=0.01$ ). B. eQTL $p$-values and LD relationship with rs17171731 in the region around FAM13B in African American subjects $\left(p=2.63 \times 10^{-5}\right)$. The grey shaded area represents the longest FAM13B gene (transcribed from left to right) in Ensembl, but the LAA transcript starts $\sim 20 \mathrm{~kb}$ proximal to rs17171731. C. Zoom in on FAM13B cis-eQTL for African American subjects (top). The SNP rs17171731 occurs in a DNAse hypersensitivity peak while the AF GWAS SNP rs2040862 does not (bottom, Roadmap Epigenomics Project data for human fetal heart). 


\section{Figure 2.}
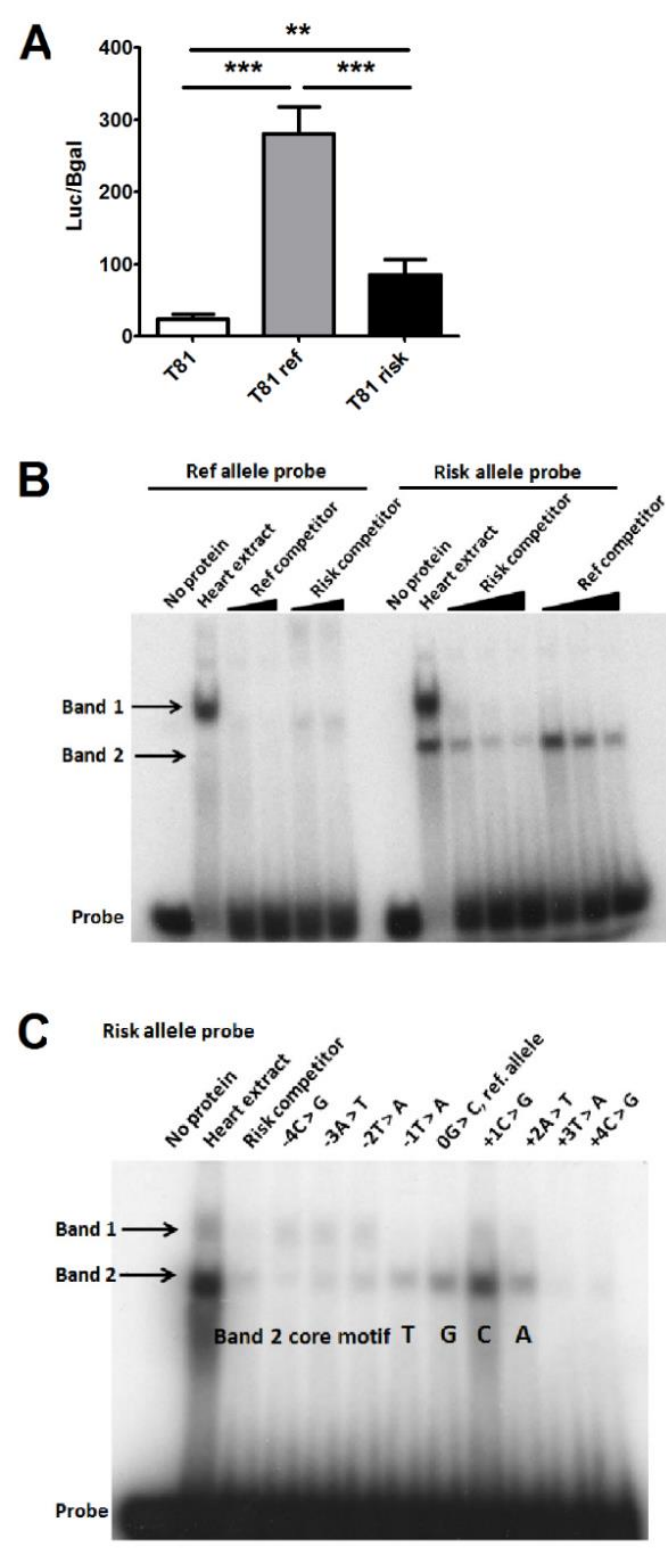

Figure 2. Functional studies for rs17171731, the candidate AF-causal SNP at chr 5q31. A. The effects of the reference and AF-risk allele of rs17171731 on luciferase expression in an enhancerless reporter plasmid in H9-derived cardiomyocytes (*** $p<0.001$ and $* * p<0.01$ by one-way ANOVA, $N=6$ ). B. Cardiomyocyte nuclear extract gel shift assay with probes for the reference and AF-risk allele of rs17171731, demonstrating that both probes shift band 1, but only the risk allele shifts band 2 . Increasing concentrations of the reference and risk allele unlabeled competitors were added as indicated $(10,25$, and 50 -fold molar excess). C. Gel shift assay of the AF-risk allele using unlabeled competitors (100-fold molar excess) containing one bp changes centered on the rs17171731 SNP, yielding the core sequence element of TGCA for the band 2 gel shift. 


\section{Figure 3.}

A

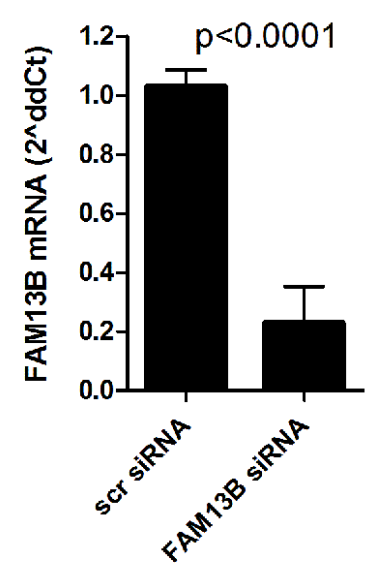

B

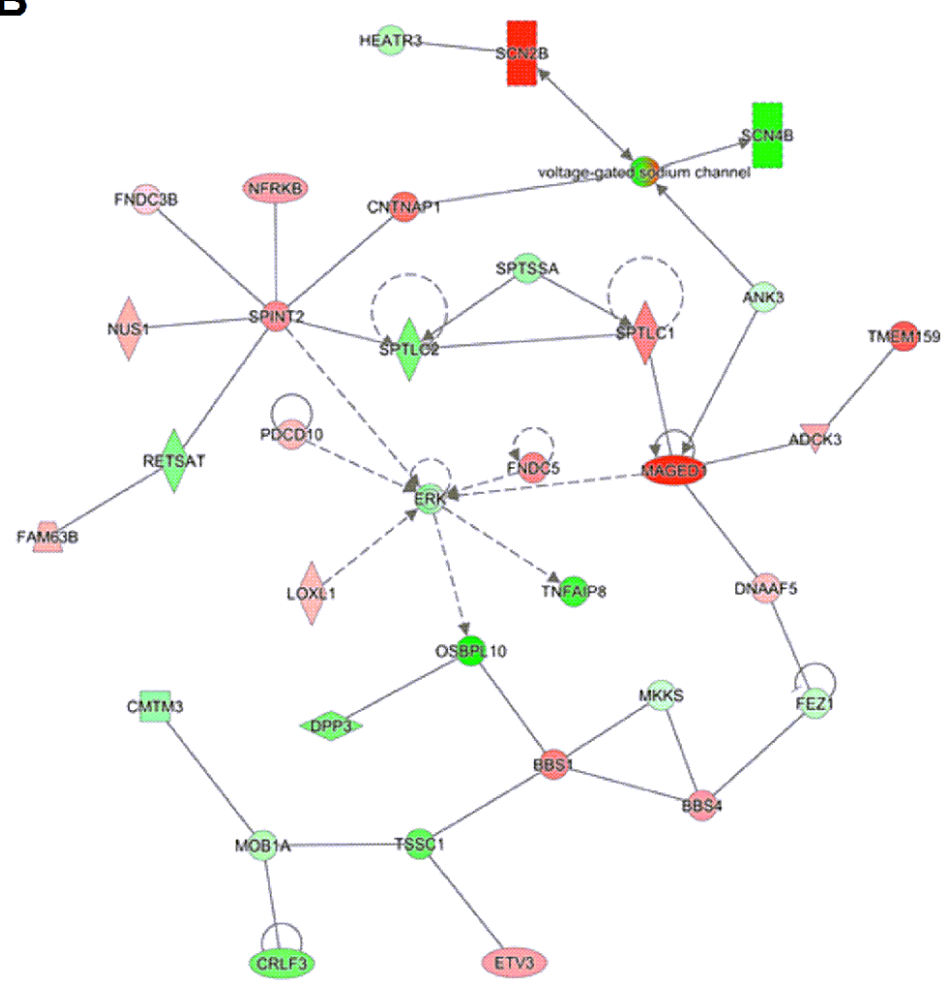

Figure 3. FAM13B Knockdown. A. qRT-PCR verification of siRNA FAM13B knockdown in iPSC-derived cardiomyocytes normalized to ACTC1 mRNA relative to the scramble siRNA (mean \pm SD, $p<0.0001$ by two-tailed t-test, $\mathrm{N}=4$ for scramble and $\mathrm{N}=5$ for knockdown from 2 independent experiments). $\mathrm{B}$. Ingenuity Pathway Analysis of gene expression changes in FAM13B knockdown vs. scramble-siRNA control. This was the second strongest empirical network identified (score $=46$ ), and it included the voltage gated sodium channel and the $S C N 2 B$ gene, the $11^{\text {th }}$ strongest regulated gene upon FAM13B knockdown. Green indicates repression and red indicates induction upon FAM13B knockdown. 


\section{Figure 4.}

A

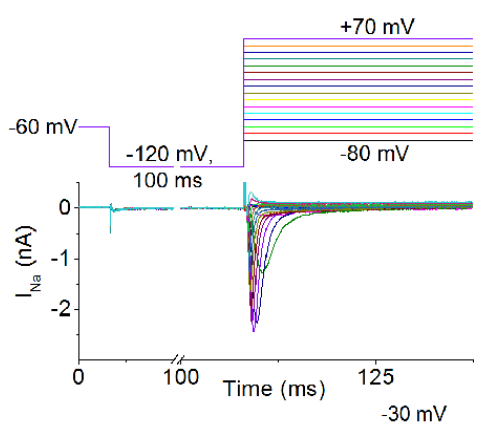

C

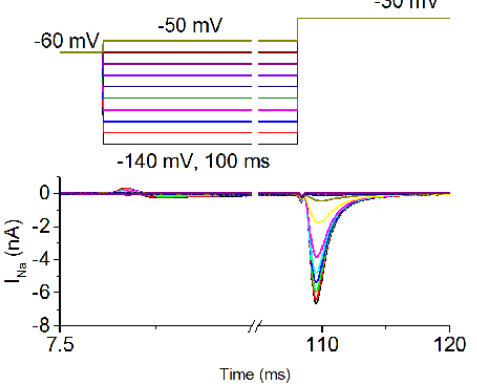

$E$

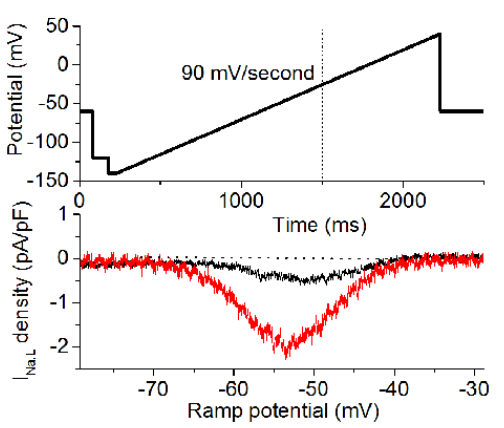

B

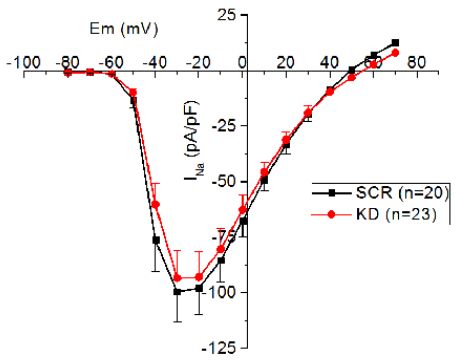

D

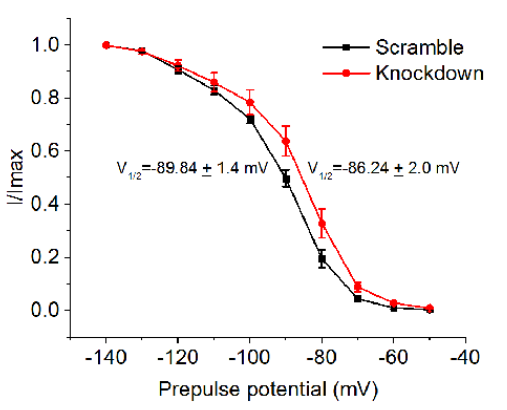

$\mathrm{F}$

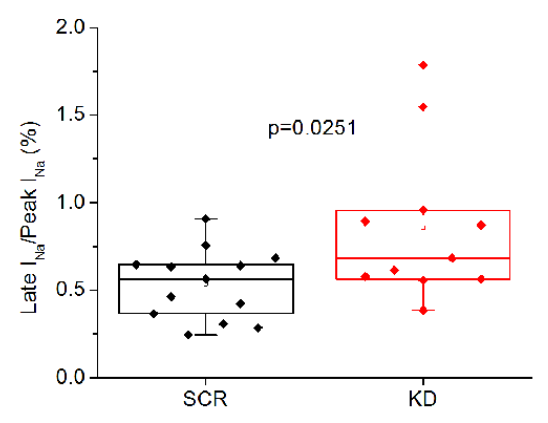

Figure 4. Impact of FAM13B knockdown on iPSC-derived cardiomyocyte sodium currents. A. Representative $I_{\mathrm{Na}}$ traces and voltage-clamp protocol. A $100 \mathrm{~ms}$ prepulse to $-120 \mathrm{mV}$ was used from a holding potential of $-60 \mathrm{mV}$. Depolarizing voltage steps from -80 to $+70 \mathrm{mV}$ were applied in $10 \mathrm{mV}$ increments. B. Summary $I_{\mathrm{Na}}-\mathrm{V}$ plot for scramble $(n=20)$ vs. FAM13B knockdown $(n=23)$ recordings. C. Inactivation protocol and representative $I_{\mathrm{Na}}$ traces. As in A, resting potential was $-60 \mathrm{mV}$, with a $100 \mathrm{~ms}$ pre-pulse used to partially recover channels from inactivation, using pre-pulse potentials from - 140 to $50 \mathrm{mV}$ in $10 \mathrm{mV}$ steps. I $I_{\mathrm{Na}}$ was recorded with a step to $-30 \mathrm{mV}$. D. $I_{\mathrm{Na}}$ currents were normalized to those recorded at $-140 \mathrm{mV}(\mathrm{I} / \mathrm{Imax})$. A Boltzmann function was used to fit the half-inactivation potential $\left(\mathrm{V}_{1 / 2}\right)$. A subset of the cells in $B$ were characterized (scramble $n=9$, knockdown $n=7$ ). E. Slowly inactivating $I_{\mathrm{Na}}$ ( $\left.I_{\mathrm{NaL}}\right)$ was recorded using a $2 \mathrm{~s}$ ramp clamp protocol from -140 to $+50 \mathrm{mV}(90 \mathrm{mV} / \mathrm{second})$. Representative current traces normalized to cell capacitance are shown over the potential range -80 to $-30 \mathrm{mV}$ for a scramble (black) and FAM13B knockdown (red) cardiomyocyte. The baseline subtracted peak negative amplitude of the current recorded in this range was used as a measure of $I_{\text {NaL. }} \mathbf{F}$. A subset of the cells in $B$ were evaluated using the protocol in $\mathbf{F}$. The percent of $I_{\text {NaL }}$ to peak $I_{N a}$ is plotted from scramble $(n=11) v s$. FAM13B knockdown $(n=13)$ cells. 


\section{Figure 5.}
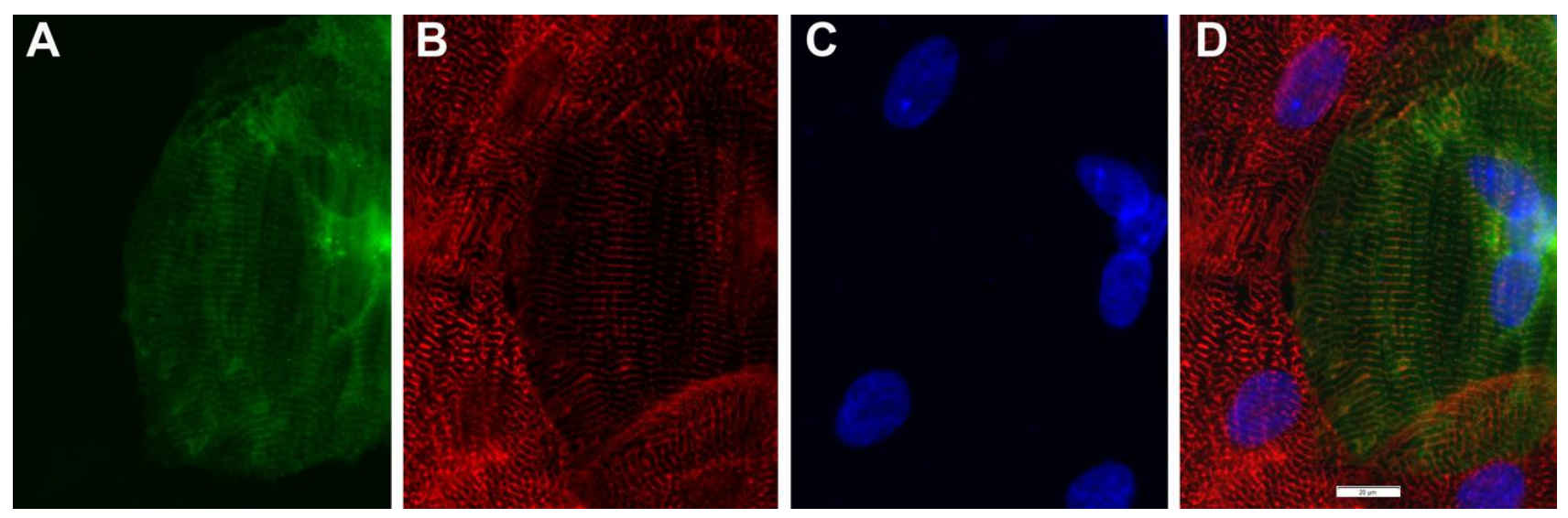

Figure 5. FAM13B-GFP fusion protein expression in iCell cardiomyocytes. A. GFP fluorescence of the fusion protein 6 days after transfection, showing expression in a sarcomere pattern and on the plasma membrane. B. $\alpha$-actinin immunofluorescence (red) showing Z-disc localization with the transfected cell surrounded by non-transfected cells. C. Hoechst 33342 staining of nuclei. D. Merged image, showing FAM13B-GFP expression overlaps with or is adjacent to the Z-disc marker protein $\alpha$-actinin. $60 \mathrm{x}$ objective lens, scale bar show $20 \mu \mathrm{m}$. 